\title{
Perceptual and motor inhibition of return: components or flavors?
}

\author{
Matthew D. Hilchey • Raymond M. Klein • Jason Ivanoff
}

Published online: 20 June 2012

(C) Psychonomic Society, Inc. 2012

\begin{abstract}
The most common evidence for inhibition of return (IOR) is the robust finding of increased response times to targets that appear at previously cued locations following a cue-target interval exceeding $\sim 300 \mathrm{~ms}$. In a variation on this paradigm, Abrams and Dobkin (Journal of Experimental Psychology: Human Perception and Performance 20:467-477, 1994b) observed that IOR was greater when measured with a saccadic response to a peripheral target than with that to a central arrow, leading to the conclusion that saccadic responses to peripheral targets comprise motoric and perceptual components (the twocomponents theory for saccadic IOR), whereas saccadic responses to a central target comprise a single motoric component. In contrast, Taylor and Klein (Journal of Experimental Psychology: Human Perception and Performance 26:1639-1656, 2000) discovered that IOR for saccadic responses was equivalent for central and peripheral targets, suggesting a single motoric effect under these conditions. Rooted in methodological differences between the studies, three possible explanations for this discrepancy can be found in the literature. Here, we demonstrate that the empirical discrepancy is rooted in the following methodological difference: Whereas Abrams and Dobkin (Journal of Experimental Psychology: Human Perception and Performance 20:467-477, 1994b) administered central arrow and peripheral onset targets in separate blocks, Taylor and Klein (Journal of Experimental Psychology: Human Perception
\end{abstract}

M. D. Hilchey $(\bowtie) \cdot$ R. M. Klein

Dalhousie University,

Halifax, Nova Scotia, Canada

e-mail: matt.hilchey@dal.ca

J. Ivanoff

Saint Mary's University,

Halifax, Nova Scotia, Canada and Performance 26:1639-1656,2000) randomly intermixed these stimuli in a single block. Our results demonstrate that (1) blocking central arrow targets fosters a spatial attentional control setting that allows for the long-lasting IOR normally generated by irrelevant peripheral cues to be filtered and (2) repeated sensory stimulation has no direct effect on the magnitude of IOR measured by saccadic responses to targets presented about $1 \mathrm{~s}$ after a peripheral cue.

Keywords Inhibition of return - Attention - Cueing - Spacebased processing $\cdot$ Eye movements: saccades $\cdot$ Strategy

\section{Introduction}

When the interval between an uninformative transient cue and target (commonly referred to as a stimulus onset asynchrony [SOA]) is short $(<300 \mathrm{~ms})$, responses to detect or localize a stimulus appearing in close spatial proximity to the cue are speeded, relative to stimuli appearing at distancematched, uncued regions. In contrast, when SOAs are in the range of $300 \mathrm{~ms}-3 \mathrm{~s}$ (Samuel \& Kat, 2003), responses to targets at cued locations are slowed, as compared with responses to targets at uncued locations. The pattern of increased response times (RTs) to cued locations at relatively long SOAs satisfies the classic conceptualization of the phenomenon inhibition of return (IOR), as discovered by Posner and Cohen (1984) and later named and explained by Posner, Rafal, Choate, and Vaughan (1985).

Since the discovery of IOR, extensive research has demonstrated the robustness of this effect, and accordingly, it has been observed reliably for ballistic eye movements (saccades) and manual keypress responses to precued targets in a rich assortment of tasks that have exploited variations on the cue-target paradigm (synonymously referred to as the 
model task or Posner cuing paradigm). One focus of these variations has been a dedicated effort (Abrams \& Dobkin, 1994b; Chica, Taylor, Lupiáñez, \& Klein, 2010; Kingstone \& Pratt, 1999; Pratt \& Neggers, 2008; Reuter-Lorenz, Jha, \& Rosenquist, 1996; Taylor \& Klein, 2000) to determine the extent to which IOR's effects on performance are primarily on the input or the output ${ }^{1}$ end of the processing continuum.

Two (additive) components of IOR's effect on saccadic responses?

Abrams and Dobkin (1994b) developed a paradigm to test whether IOR comprises inhibitory components at both the input and output stages of processing when saccadic responses are made. They compared two conditions in which, after ignoring an uninformative peripheral cue, participants were required to make an eye movement to the target dot presented in a peripheral box to the left or right of fixation. In one condition the target was, like the cue, a peripheral onset. The key innovation was that in the other condition, the target was a centrally presented arrow. The task in this case was for the participants to shift their gaze in the direction of the arrow toward the indicated peripheral box. The rationale behind this manipulation was relatively straightforward. Because, on cued trials, the cue and target would occupy the same location in the peripheral target condition, there was a possibility that (1) the cue would increase saccadic reaction time (SRT) through an effect on the input end of the processing continuum, (e.g., by degrading or delaying the processing of the subsequent peripheral target when presented in the same location at the cue) and/or (2) the cue would increase SRT through an effect on the output end of the processing continuum (e.g., by a bias against responding in the cued direction). In the central target condition, by contrast, the target appeared at a location that would not have been stimulated by the uninformative peripheral cue; consequently, there would be no possibility that repeated stimulation at a peripheral location could degrade or delay subsequent information processing (the input view). A delay in responding to a central arrow that points in the direction of the uninformative peripheral cue can, however, be attributed to IOR operating

\footnotetext{
${ }^{1}$ Abrams and Dobkin (1994b) and Taylor and Klein (2000) use different terms to refer to the input-related and output-related processes that may be operating when IOR is observed. On the input side, they refer to "processes involved in detecting visual stimuli" versus "slowed visual processing," respectively, with Taylor and Klein (2000) acknowledging that "slowed visual processing" could be a delay in detection or in the reorienting of attention. On the output side they refer to "inhibition . . . related to the movement of the eye" versus "slowed motor production," respectively. We will use the neutral terms input and output to refer to this broad distinction. There has been considerable speculation, but no specific determination yet, of the precise mechanism of operation of these two effects.
}

after input processing on decision or motoric processes (the output view).

From this reasoning, concrete behavioral predictions can be derived (see Fig. 1). If there is a single input effect, centrally presented targets will show no IOR, whereas peripherally presented targets will $[\mathrm{IOR}($ central target $)=0$ and IOR(peripheral target) $>0$ ]. If there is a single output effect, the magnitude of IOR will be equivalent for peripherally and centrally presented target [IOR(peripheral target) $=$ IOR (central target)] so long as they entail the same response. If there are two unique components to IOR, the magnitude of behavioral IOR should be greatest in the condition in which both components contribute to the effect, relative to the condition in which only one component acts on RTs [IOR (peripheral target) $>$ IOR(central target)]. In line with the latter prediction, Abrams and Dobkin (1994b) found that the magnitude of IOR was $\sim 10 \mathrm{~ms}$ in the block of trials with central arrow targets, whereas the magnitude of IOR was $\sim 25 \mathrm{~ms}$ in the block of trials with peripheral targets, a difference that was statistically significant. From this pattern of results, Abrams and Dobkin (1994b) concluded "that some of the inhibition is due to processes involved in detecting visual stimuli, and some of the inhibition is related to the movement of the eye." Abrams and Dobkin's (1994b) article is one of the most influential empirical studies in the literature on IOR and is often cited to support the notion that saccadic responses to targets in IOR paradigms are affected by separate input- and output-related inhibitory components.

Two "flavors" of IOR, only one of which operates with saccadic responses?

Whereas Abrams and Dobkin (1994b) presented evidence for two additive components of IOR, their pattern of results was not replicated in a similarly influential article by Taylor and Klein (2000) in an experiment (Experiment 4) in which central arrow and peripheral target conditions were intermixed. Both studies had comparable SOAs $(960 \mathrm{~ms}$, as compared with 1,000 ms in Taylor \& Klein, 2000), both studies adopted the convention of using a cue back at fixation, and both studies entailed ignoring a noninformative peripheral cue. Whereas Abrams and Dobkin (1994b) demonstrated that the magnitude of IOR was $\sim 15 \mathrm{~ms}$ greater in the peripheral target condition, Taylor and Klein (2000) demonstrated that the magnitude of IOR was equivalent between the two conditions. The empirical discrepancy is illustrated in Fig. 2.

Taylor and Klein (2000) interpreted their findings in the context of a comprehensive investigation of 24 conditions (including the conditions from Abrams \& Dobkin, 1994b). The pattern of results for these 24 conditions is illustrated in Fig. 3. 
Fig. 1 Illustration of the behavioral predictions for the attentional/perceptual, motoric, and two-components accounts

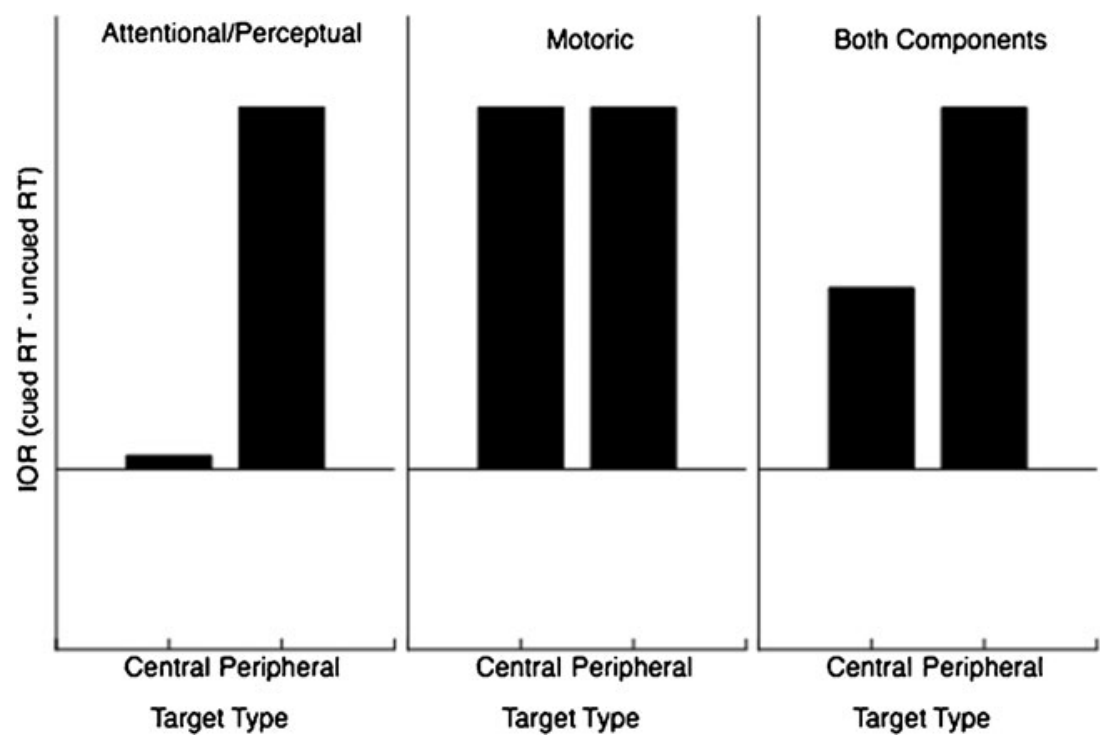

A neglected empirical discrepancy with theoretical implications

What can be gleaned from this discussion is that the conflicting empirical results between Abrams and Dobkin (1994b) and Taylor and Klein (2000) entail two different theoretical implications for IOR when measured about $1 \mathrm{~s}$ after an ignored, uninformative peripheral cue. In essence, one pattern of results (e.g., Taylor \& Klein, 2000, Experiment 4; see Fig. 2, right panel, in the present article) suggests a single effect operating on output stages or processing when IOR is measured by a saccadic response, whereas the other pattern of results (e.g., Abrams \& Dobkin, 1994b, Experiment 2; see Fig. 2, left panel, in the present article) suggests a two-components view (i.e., the IOR measured by saccades to peripheral targets is composed of effects operating simultaneously at both input and output stages of processing).
Fig. 2 Results from Experiment 2 in Abrams and Dobkin (1994b), on the left, and from Experiment 4 in Taylor and Klein (2000), on the right
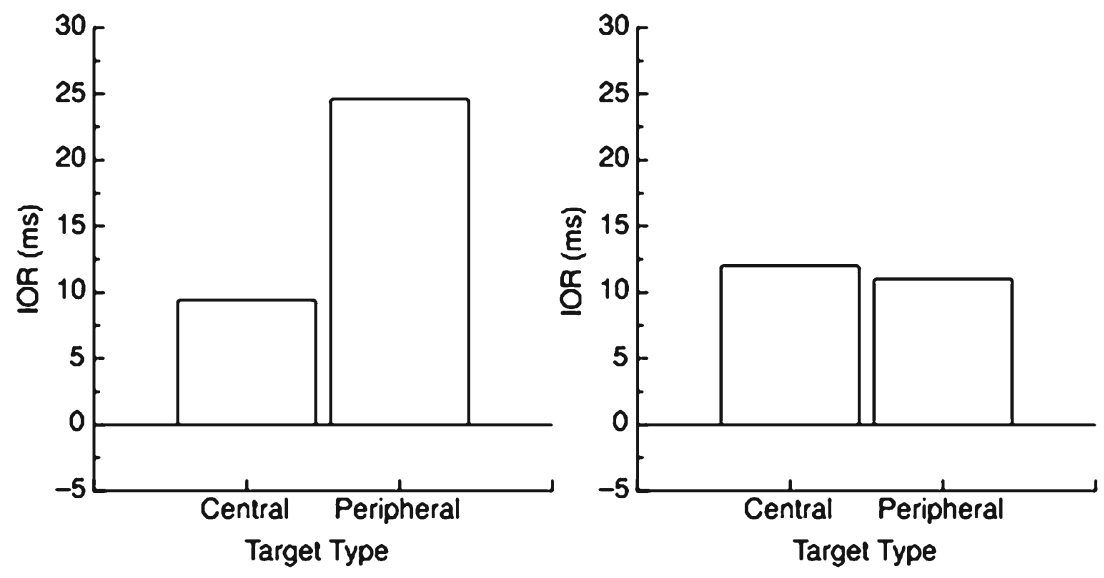


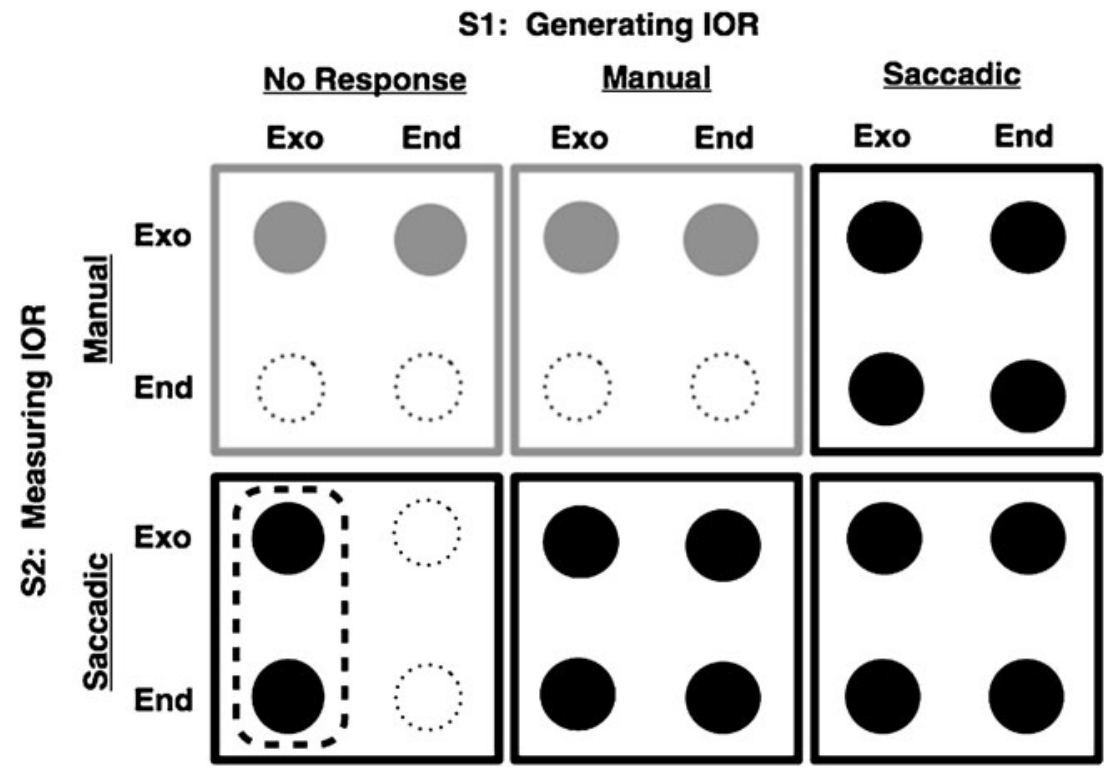

Fig. 3 All possible cue-target/target-target and response configurations experienced by all of the participants in Taylor and Klein's (2000) comprehensive investigation into the causes and effects of IOR. Exogenous ("Exo") and Endogenous ("End") signals (S1 = first signal; S2 = second signal) represent peripheral and central (arrow) stimuli, respectively. The six boxes represent the conditions that were randomly intermixed in a session. The type of responses (no response, manual, or saccadic) to the two signals is underlined. The conditions for which IOR (cue RT > uncued RT) was observed are shown as filled circles;

Considering how different these interpretations are and the results that were used to endorse them (see Fig. 2), it is surprising how infrequently the discrepancy has been noted. Since Taylor and Klein (2000) pointed out the discrepancy, no studies have suggested an explanation, and only one has mentioned it ( $\mathrm{Li} \&$ Lin, 2002) until Klein and Hilchey's (2011) recent review of oculomotor IOR. Notably, whereas there is a fundamental difference between the two interpretations, each allows for IOR to have effects at the input and output levels of processing. The important difference is that in the no-response saccade condition Taylor and Klein (2000) assert that inhibition is operating only at the output level of processing, while in this same condition, Abrams and Dobkin (1994b) assert that inhibition is operating at both levels of processing simultaneously.

Three explanations for the empirical discrepancy

In an effort to reconcile the empirical discrepancy (with its consequent theoretical implications) between these two investigations, the methodologies were contrasted. Critically, these studies diverged conspicuously on several methodological dimensions. Here, we consider three key methodological differences or features that we will refer to as fixation removal confound, perceptual confusion, and blocking. the unfilled dotted circles indicate conditions for which IOR was not observed. The gray versus black boxes and disks represent the two flavors of IOR, which are distinguished by whether (output flavor) or not (input flavor), when it was observed with a peripheral S2, IOR was also observed with a central arrow S2. The dashed oblong highlights the conditions for which Abrams and Dobkin (1994b) and Taylor and Klein (2000) reported conflicting findings, the discrepancy that is the focus of the present study

First, in Abrams and Dobkin (1994b), for peripheral, but not for central targets, the removal of fixation coincided with target onset. This confound was not present in Taylor and Klein (2000). As was noted by Taylor and Klein (2000), who focused their attention on the different magnitudes of peripheral cue-induced IOR in the two studies (see Fig. 2), in a different 1994 article, Abrams and Dobkin (1994a) reported that removal of fixation increased IOR. Thus, the increased IOR with peripheral cues in Abrams and Dobkin's (1994b) study might not have been due to an effect of IOR upon input levels of processing; rather, it might simply have been produced by removal of the fixation stimulus with peripheral cues, a removal that was not present in their central cue condition.

In their effort to explain a different empirical discrepancy (does fixation removal increase or decrease IOR?; see footnote 2), Hunt and Kingstone (2003) described a methodological feature of Abrams and Dobkin (1994a) that was replicated by Abrams and Dobkin (1994b). They suggested that

Abrams and Dobkin's methods were very different from the standard and simple cuing paradigm normally used to generate IOR. Abrams and Dobkin used a peripheral asterisk as a cue, and then the fixation stimulus turned from a circle to an asterisk that was identical to the peripheral cue and then back to a 
circle, and then the target, a circle, which was identical to the fixation circle, appeared either in the same location as the peripheral asterisk or in the opposite location. The potential for perceptual confusion in this experiment was substantial because the identity of the cue, fixation, and target overlapped within a single trial. If participants were using strategies to cope with the perceptually confusing display, it could produce idiosyncratic results. (p. 1073)

Because this description applies equally to the methods used by Abrams and Dobkin (1994b) and does not apply to Taylor and Klein's (2000) methods, perceptual confusion offers a second possible source of the empirical discrepancy.

Finally, Abrams and Dobkin (1994b) administered the central arrow and peripheral target conditions in separate blocks, whereas these conditions were intermixed in a single block in Taylor and Klein (2000). Minimizing variation of conditions within a block of trials is a respectable method for allowing observers to engage a mental set that might optimize performance (for a heroic effort in this regard, see Hecht, Schlaer, \& Pirenne, 1942). On the other hand, separating two or more levels of a factor into separate blocks is one way to explore how observers might adopt different strategies in response to the different conditions. When a scientist is interested in understanding how the observer's information-processing machinery is operating unconfound$e d$ by different strategies that might be adopted in advance of the key manipulation, it is essential to ensure that the observer's mental state before the events of a trial begin be equated. This can be ensured only by randomly intermixing the conditions of interest (see Klein, 1977, Experiment 2, for one use of blocking vs. mixing to reveal that visual dominance over kinesthetic inputs is likely due to a strategic bias to attend vision; and see Pratt, Sekuler, \& McAuliffe, 2001, for their use of the same kind of comparison to confirm that the finding of contingent capture by Folk, Remington, \& Johnston, 1992, was due to the proposed strategic focusing of attention on the feature defining the target in this seminal study, and not simply due to the cue-target similarity). In their well-known and insightful critique of the use of the "neutral" condition in a Posner cuing task, Jonides and Mack (1984) offerred a similar warning about a blocked design: "Guarding against some conditions, such as those that may be induced by a blocked presentation of cues, may merely require some thought in experimental design. Others may be more insidious" (p. 35). This warning applies equally to the blocked presentation of targets.

Recognizing the untowardness of Abrams and Dobkin's (1994b) blocked design, Klein and Hilchey (2011; see also Hunt \& Kingstone, 2003) suggested that their finding (peripheral IOR was greater than central IOR) was generated by untoward differences in mental set made possible by their collecting data from arrow and peripheral targets in separate blocks. When the target was never in the periphery (always an arrow at fixation), the participant could adopt an attentional control setting that encouraged effective filtering of the irrelevant peripheral cues. This would not be possible in a block when all (Abrams and Dobkin's peripheral target blocks) or some (Taylor \& Klein, 2000) of the targets were peripheral. (p. 480)

Subsumed under the label attentional control settings (ACSs), there is a considerable literature that explores the role of blocking-induced expectancies (both spatial and nonspatial) about the upcoming target. This evidence converges on the view that capture of attention by a peripheral target may be contingent upon the nonspatial (as demonstrated in Folk et al., 1992, seminal article) and spatial (e.g., Ishigami, Klein, \& Christie, 2009; Theeuwes, 1991; Yantis \& Jonides, 1990) control settings adopted by the observed to prepare for the possible targets. The flexibility of spatial attention to respond to spatial expectancies and task demands has given rise to a variety of mechanism-suggesting metaphors: Eriksen's "zoom lens" (Eriksen \& St. James, 1986), Laberge's "attentional range" (Laberge, 1983), Goldsmith and Yeari's (2003) "attentional focussing," and Theeuwes's (1994) "attentional window."

Until recently it was thought that IOR might be immune to such attentional control because, when the (nonspatial) feature-based methods of Folk et al. (1992) were repeated using a cue-target SOA long enough to elicit IOR, IOR was not observed following cues that matched the observer's target-based ACS for color (Gibson \& Amelio, 2000; Pratt $\&$ McAuliffe, 2002; Pratt et al., 2001), even though these cues did generate attentional capture at early SOAs. Some studies have suggested that the time course and possibly magnitude of IOR might be dependent on attentional control settings related to the nature of the task (e.g., the likelihood that the target is accompanied by a distractor; cf. Lupiáñez $\&$ Milliken, 1999). Following Klein's $(2000,2005)$ proposal, we believe that these effects have more to do with the speed and/or probability of attentional disengagement from the cue than with the generation of IOR per se. Recently, however, it has been demonstrated that IOR can be contingent on the degree to which attention is focused on fixation around the time of the peripheral cue (Wang \& Klein, 2012). This location-based ACS finding provides a more directly relevant empirical foundation for us to hypothesize that in the blocked design of Abrams and Dobkin (1994b), IOR measured in a block with central targets might be less than that in a block with peripheral targets because of different spatial ACSs adopted by the observers in the two blocks, 
rather than because of the locations of these imperative signals.

In Taylor and Klein's (2000) experiment, for which a block of trials comprised both centrally and peripherally presented targets, the participant's mental state at the time of the presentation of a target could not have been "tuned" to the central versus peripheral nature of the upcoming target, because the two types of target were randomly intermixed. Thus, given that half of the targets appeared in the periphery in a mixed (balanced) design, it is more reasonable to assume that the observers spread attentional resources diffusely throughout the target display such that all targetrelevant space (i.e., where targets often appeared spatially) was attended to some degree (Goldsmith \& Yeari, 2003).

An experimental test of these explanations

We first attempted to closely replicate the trial structure from Abrams and Dobkin (1994b) while randomly assigning participants to a session with only central or only peripheral target stimuli. We subsequently maintained this trial structure while intermixing the two target conditions, as did Taylor and Klein (2000). A comparison between a mixed and blocked design allows for the following predictions: If the increased IOR for peripheral relative to central targets in the blocked conditions was attributable to a centrally focused ACS, the magnitude of IOR would be greater in the blocked peripheral target condition, as compared with the blocked central target condition, but equivalent when intermixed. Such a result-implying an unanticipated ACS spurred on by the confinement of targets to fixationwould severely undermine one of the central pillars of the two-components theory of IOR while endorsing Taylor and Klein's (2000) proposal that when the saccadic system is engaged during a task, the effects of IOR are purely at output levels of processing. On the other hand, if the Abrams and Dobkin (1994b) pattern of results was attributable to perceptual confusion or the fixation removal confound - in other words, if Klein and Hilchey's (2011) ACS proposal were wrong - the pattern of results from Abrams and Dobkin (1994b) would be replicated in both the blocked and mixed designs. In that case, further experimentation would be required to determine whether perceptual confusion or the stepeffect confound were responsible for the discrepancy or whether the pattern reported by Taylor and Klein (2000) was a fluke. Anticipating the results, in Experiment 1, we obtained evidence supporting the ACS explanation for the discrepancy and, consequently, the two-flavors view. Then, to address our concern as to whether fixation removal was modulating the magnitude of IOR for peripheral targets, in Experiment 2 we reran the mixed target condition with a sustained fixation stimulus to determine whether differences between central and peripheral targets could be achieved in a paradigm devoid of the two aforementioned confounds. Again, IOR was identical for central and peripheral targets, supporting the operation of only the motoric flavor of IOR in this task.

\section{Experiment 1}

In this experiment, the stimulus event used to generate IOR was a to-be-ignored peripheral onset. The peripheral target or centrally presented arrow target instructed participants to make a saccadic eye movement to either the placeholder in which a target appeared or to the placeholder to which an arrow pointed, respectively. To test whether the increase in IOR that Abrams and Dobkin (1994b) reported for the peripheral target condition, relative to the central target condition, resulted from two additive components or was an artifact spurred on by different ACSs in the blocked design, we closely replicated the sequence of events in their methodology while manipulating whether the peripheral and central targets were blocked (as in Abrams \& Dobkin, 1994b) or mixed (as in Taylor \& Klein, 2000). It must also be noted that unlike in Abrams and Dobkin (1994b), our blocked target condition (peripheral or central) was a between-subjects variable. We specifically chose this design feature to avoid any possibility of carryover of attentional strategies from one block to the next. We fully expected to replicate the finding that the magnitude of IOR for saccadic responses to peripheral targets would be greater than the magnitude of IOR for saccadic responses to central targets when these were blocked. If this difference in IOR scores were attributable to distinct attentional deployment strategies for central and peripheral target blocks, intermixing peripheral and central targets would eliminate any numerical difference between these two target types. If perceptual confusion (Hunt \& Kingstone, 2003) or the fixation removal (Taylor \& Klein, 2000) contributed primarily to these differences, then, because we adhered to the trial sequences used by Abrams and Dobkin (1994a, 1994b), the pattern of results in the mixed and blocked conditions should be similar.

\section{Method}

Participants Eighteen undergraduate students (11 females and 7 males) from Dalhousie University participated in a single 75- to 90 -min session for course credit or monetary compensation $(\$ 15 \mathrm{CDN})$. All participants were naive as to the purposes of the experiment and reported either normal or corrected-to-normal vision. Two participants were lefthanded (both of whom participated in the blocked peripheral target condition). 
Apparatus and procedure All participants were tested in a dimly lit room, and all stimuli were presented against a black background. Stimuli were presented on a 19-in. ViewSonic Optiquest Q95 CRT monitor (Q95-3) connected to an Intel Core Duo processor, at a viewing distance of 22.5 in. An Eyelink II eye-monitoring system was used to record the eye movements of the participant from the initiation of a drift correction phase, which ensured the accuracy of the calibration parameters at the beginning of the trial, until a saccadic response was executed upon target presentation. The EyeLink headset was connected to a host computer, operating on a Pentium Intel 4 processor, which projected online gaze coordinates to a secondary monitor that was obscured by a black curtain during experimentation. After participants performed a 9-point calibration procedure to determine and validate the precision of the eye tracker within a half degree of visual angle, the host computer provided accurate information about gaze position approximately once every $4 \mathrm{~ms}$.

See Fig. 4 for the sequence of events. At the beginning of every trial, a red fixation cross $\left(0.5^{\circ} \times 0.5^{\circ}\right)$ appeared at the
Euclidean midpoint of an imaginary horizontal line segment between two placeholder boxes $\left(0.8^{\circ} \times 0.8^{\circ}\right)$. White placeholders were positioned equidistantly from the fixation cross at $7^{\circ}$ to the left and right. At the beginning of every trial, a manual space bar response was required when the observer's subjective experience was that of having successfully fixated the fixation cross. If this drift correction phase was completed successfully, the fixation cross changed from red to white, providing a clear demarcation between the stage that reassessed the validity of the calibration procedure and the subsequent experimental phase of the trial. Otherwise, the fixation cross remained red, and additional manual responses were made until the central gaze position was accepted by the eye tracker.

If the trial was successfully initiated, the white fixation cross appeared onscreen for $3 \mathrm{~s}$. After this point, the methods mirrored those in Abrams and Dobkin (1994b). The white fixation cross transformed into a white circle $\left(\right.$ diameter $=0.5^{\circ}$ ), which remained onscreen for $800 \mathrm{~ms}$. The cue, a noninformative peripheral asterisk (diameter $=0.5^{\circ}$ ), then appeared randomly in one of
Fig. 4 Sequence of events from Experiments 1 and 2. Note that the fixation stimulus was never extinguished in Experiment 2. See the text for additional details

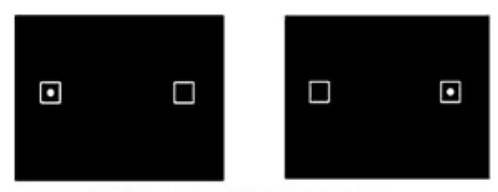

Until saccadic response

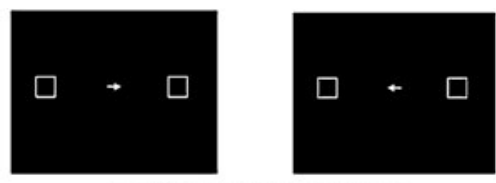

Until saccadic response

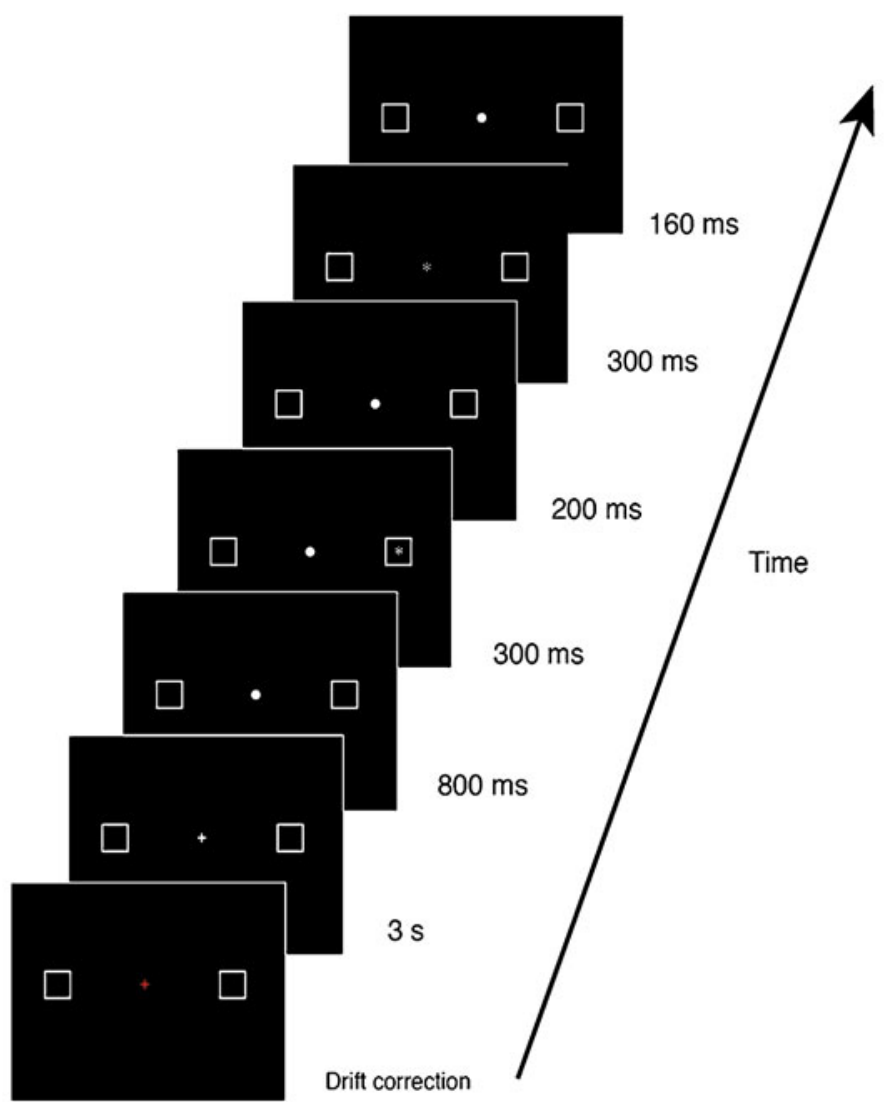


the two placeholders for $300 \mathrm{~ms}$. Participants were explicitly instructed that the cue was irrelevant in that it was completely unpredictive of the location of the second imperative peripheral onset and/or of the direction of the imperative central arrow. Two hundred milliseconds following the transient peripheral event, the fixation symbol was replaced by an asterisk, a symbol that, it was explained to participants, could inform them that an imperative stimulus would be appearing soon thereafter, for $300 \mathrm{~ms}$, and then by a white circle for $160 \mathrm{~ms}$. In the blocked central target condition, the white circle was replaced by a central leftward- or rightwardpointing target arrow (measuring $1^{\circ}$ of visual angle in width) for which a speeded saccade was required to the placeholder corresponding to the direction of the arrow. In the blocked peripheral target condition, the onset of a white peripheral circle coincided with the removal of fixation. In this case, a speeded saccade was required to the location of the peripheral onset target. In the mixed target condition, the two abovementioned target conditions and task demands were randomly intermixed. After a saccade had been executed or, alternatively, after $1.5 \mathrm{~s}$, the trial ended, and $3 \mathrm{~s}$ later the next trial was initialized.

Eye movement monitoring The calibration of the eyemonitoring system was accepted if the subsequent validation procedure obtained an average accuracy less than or equal to $0.5^{\circ}$ of visual angle. As aforementioned, a drift correction was conducted on a trial-by-trial basis to maintain the accuracy of the calibration procedure. In the rare event that the drift correction procedure failed, the calibration process was repeated, the remaining trials were reshuffled, and participants returned to the block of trials in which they were performing. Every two blocks (64 experimental trials), eye monitoring was recalibrated.

Throughout a trial, eye movements, rather than being monitored selectively after the offset of the cue and immediately before the presentation of a target (Abrams \& Dobkin, 1994b), were continuously monitored (at a sampling rate of approximately once every $4 \mathrm{~ms}$ ). If gaze position from fixation exceeded $3^{\circ}$ of visual angle at any point in time before the presentation of a target, the present trial abruptly ended and was reshuffled among the remaining trials. The velocity threshold to detect a saccade was set to $35^{\circ} \%$, and the SRTs were computed to be the interval between target presentation and the initiation of the saccade.

Design Participants were randomly assigned to one of three conditions (central arrow targets, peripheral onset targets, and mixed central arrow and peripheral onset targets conditions), yielding three groups of 6 participants each. They performed 10 blocks of 32 trials each, the first of which was discarded as practice. In all cases, the location of the first peripheral onset was random and uninformative about target location or the direction of the central arrow target. In the mixed and central arrow target blocks, the central directional arrow signaled a leftward or rightward saccadic response. In the mixed and peripheral onset target blocks, the onset appeared randomly either left or right of fixation and signaled a prosaccadic response.

\section{Results}

In all conditions, the first block of data was excluded from analyses as practice. In the blocked central, blocked peripheral, and mixed target conditions, $3.9 \%, 1.8 \%$, and $7.3 \%$ of the trials, respectively, were terminated abruptly because gaze position was outside the fixation criterion before target onset. Again, because these trials contained no information about saccadic responses to target onsets, these trials were excluded from analyses. After these exclusions, there were 1,728 total trials in the peripheral and mixed target conditions. In the central target condition, there were 1,708 trials because of an untimely power outage resulting in a loss of data in block 8 for 1 participant.

Of the remaining trials, following Abrams and Dobkin (1994b), trials on which a saccade was made in the direction opposite to the peripheral onset or opposite to the direction of the central arrow were excluded from the SRT analysis. The error rates were low in all conditions, resulting in the exclusion of $<2 \%$ of trials for the SRT analyses. The threshold for anticipatory saccades excluded all SRTs less than or equal to $100 \mathrm{~ms}$ (Kingstone \& Klein, 1993). Furthermore, we excluded all trials on which the SRTs exceeded $1 \mathrm{~s}$, reasoning that these SRTs were unlikely to be measuring the psychological processes underlying IOR. Saccadic anticipations and SRTs in excess of $1 \mathrm{~s}$ were rare in all conditions (combining for $<1 \%$ of trials in each condition).

SRTs and error rates are shown in Table 1. Analyses of the error rates revealed no significant differences. The SRTs from the blocked conditions were submitted to a 2 (cued or uncued target) $\times 2$ (peripheral or central target block) mixed analysis of variance (ANOVA, where target type was a between-subjects factor). This revealed a main effect of cuing, $F(1,10)=6.26, p<.05$, that was qualified by an interaction between cuing and target type, $F(1,10)=$ $7.39, p<.05$. Clearly, this interaction arose because, as can be seen in Fig. 5 (left panel), IOR was obtained in the blocked peripheral target condition $(M=34.25 \mathrm{~ms})$, but not in the blocked central target condition $(M=-1.41 \mathrm{~ms})$. The difference between cued and uncued SRTs resulted in greater IOR in the blocked peripheral target condition, relative to the blocked central target condition, where there was neither numerical nor statistical evidence for IOR. The SRTs from the mixed target condition were submitted to a similar 2 (cued or uncued target) $\times 2$ (peripheral or central target type) repeated measures ANOVA. SRTs were shorter for peripheral 
Table 1 Composite saccadic response time (SRT) data for the IOR scores and the error rates for all experiments

\begin{tabular}{llllll}
\hline Experiment $\#$ & Design & Fixation Removal? & Target Type & Cued SRT in ms (Error \%) & Uncued SRT in (Error \%) \\
\hline 1 & Blocked & No & Central Arrow & $299.61(0.00 \%)$ & $301.02(0.23 \%)$ \\
1 & Blocked & Yes & Peripheral Onset & $270.31(0.00 \%)$ & $236.06(0.23 \%)$ \\
1 & Mixed & No & Central Arrow & $293.94(3.34 \%)$ & $265.84(1.16 \%)$ \\
1 & Mixed & Yes & Peripheral Onset & $215.60(0.48 \%)$ & $194.91(0.00 \%)$ \\
2 & Mixed & No & Central Arrow & $306.97(4.50 \%)$ & $279.76(1.20 \%)$ \\
2 & Mixed & No & Peripheral Onset & $249.67(0.23 \%)$ & $221.49(0.00 \%)$ \\
\hline
\end{tabular}

Note. "Fixation removal?" refers specifically to whether the fixation stimulus was extinguished at the time of target onset

targets relative to central targets, $F(1,5)=20.56, p<.05$, and were longer for cued locations as compared with uncued locations, $F(1,5)=17.96, p<.05$, revealing IOR (see Fig. 5, middle panel). Importantly, there was no interaction between target type and cuing, $F<1$, indicating that there was no statistical difference in the IOR scores for centrally $(M=$ $28.10 \mathrm{~ms})$ and peripherally $(M=20.69 \mathrm{~ms})$ presented targets.

\section{Discussion}

By design, the stimuli and procedure for the blocked central and peripheral target conditions in Experiment 1 were a close replication of those in Abrams and Dobkin (1994b). Empirically, however, and unlike Abrams and Dobkin (1994b), we found no evidence of IOR in the central target condition. To be sure, however, the magnitude of IOR in blocked central target conditions is typically small (e.g., Rafal, Egly, \& Rhodes, 1994) or nonexistent (e.g., Li \& Lin, 2002). As was noted earlier, we used a completely between-subjects blocked design, while Abrams and Dobkin's (1994b) participants were tested in both central and peripheral target blocks, with the order counterbalanced. A likely contributor to our finding of no IOR with blocked central targets and Abrams and Dobkin's (1994b) finding of $10 \mathrm{~ms}$ of IOR is that none of our participants would have experienced a carryover effect from having attended to the periphery when they were subjected to the central target block. Regardless of the degree to which such carryover effects might have contributed to Abrams and Dobkin's (1994b) finding of $10 \mathrm{~ms}$ of central target IOR, the present results converge with the findings of $\mathrm{Li}$ and Lin and point to the possibility that some observers might excel at filtering out the task-irrelevant peripheral cues when targets are never presented in the periphery.

More importantly, when these conditions were intermixed, there was no statistical or numerical evidence that the magnitude of IOR was greater for peripheral than for central targets. Moreover, there was robust evidence for IOR with both centrally and peripherally presented targets. Given that the only difference between these conditions (blocked and mixed) is the extent to which one might

a Blocked Targets (Exp. 1)

b Mixed Targets (Exp. 1)

C Mixed Targets (Exp. 2)
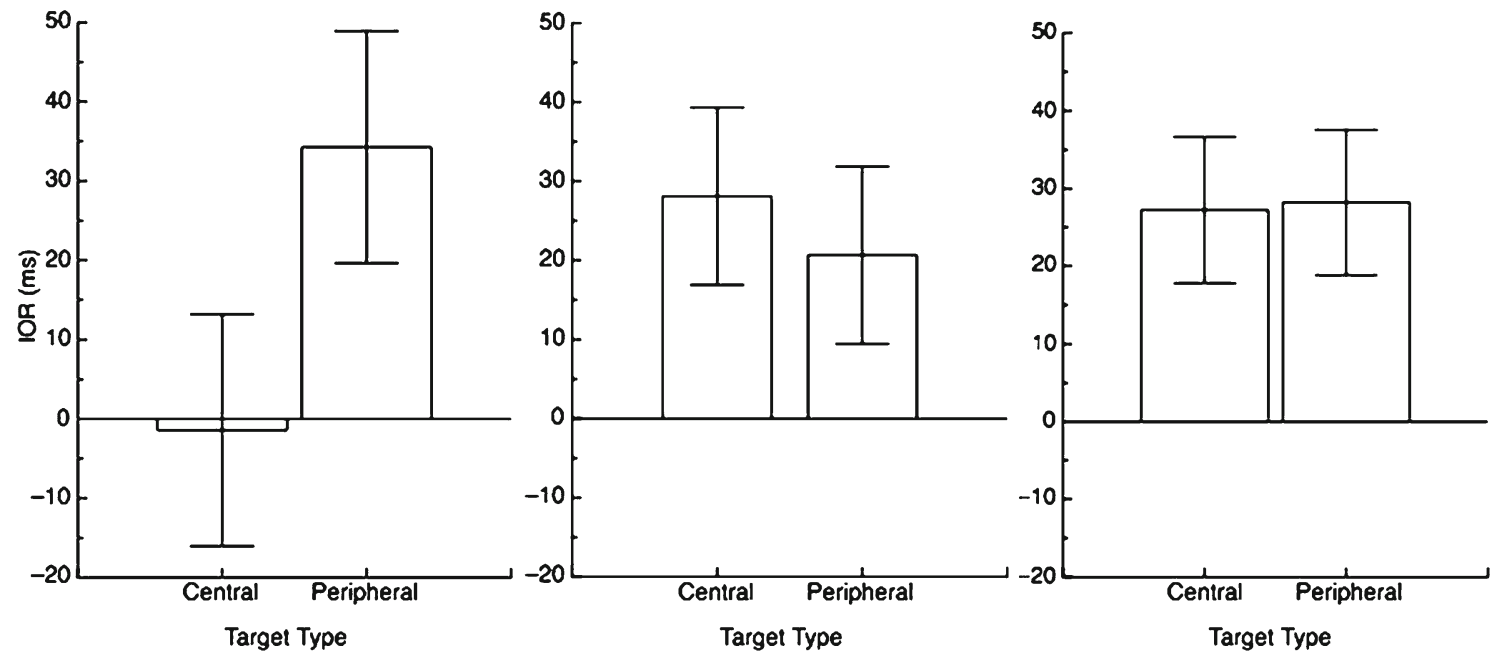

Fig. 5 Magnitude of IOR for central and peripheral targets in each experiment. a From the blocked design of Experiment 1. b From the mixed design of Experiment 1. c From the mixed design of Experiment 2. Error bars are Fisher's LSD 
expect a target at the center or in the periphery, it is likely that spatial expectancies about the locations of targets affect the spatial distribution of attentional resources such that the peripheral cues receive less processing in a block when all targets are presented at fixation (central arrow target trials) than when all (a block of peripheral targets) or half (a mixed block of central and peripheral targets) of the trials are in the periphery.

We must remind the reader that it was by design that Experiment 1 included a fixation removal confound (removal characterized the peripheral but not the central condition). Whereas this confound does not challenge the different pattern of results we obtained in the blocked versus mixed conditions, one may wonder whether the removal of fixation might have reduced the measured magnitude of IOR for peripherally presented targets in our mixed condition, thus obscuring a pattern of results that would be consistent with the two-components view. This possibility can be entertained, despite Abrams and Dobkins (1994a) finding of increased IOR following fixation removal (see also Guimaraes-Silva, Gawryszewski, Portugal, \& Klausner-de-Oliveira, 2004), because other researchers (Hunt \& Kingstone, 2003; Souto \& Kerzel, 2009) have reported the opposite finding (that fixation removal can decrease IOR). Because the possible contribution of fixation removal in Experiment 1 is, on the basis of the literature, ambiguous, Experiment 2 was conducted to determine whether IOR would continue to be equivalent for peripherally and centrally presented targets in a mixed design when the fixation removal confound was eliminated (a fixation stimulus remains onscreen throughout the trial on both peripheral and central trials).

\section{Experiment 2}

In Experiment 2, we replicated the mixed block of Experiment 1 without removing fixation in either the central or peripheral target condition. If peripheral onset targets comprise a second attentional/perceptual component, they will show more IOR than will central targets.

\section{Method}

Participants Thirteen new undergraduate students (9 females and 4 males) from Dalhousie University participated in a single 75- to 90-min session for course credit or monetary compensation $(\$ 15 \mathrm{CDN})$. All participants were naive as to the purposes of the experiment and reported either normal or corrected-to-normal vision. All but 1 participants were right-handed.
Apparatus and procedure Experiment 2 was identical to Experiment 1, except that only the mixed target condition was administered to participants and, in the peripheral target condition, the fixation stimulus was not removed. Consequently, in Experiment 2, there was something at fixation at the time of the target delivery (following Taylor \& Klein, 2000), regardless whether the target was central or peripheral.

\section{Results}

The first block of data was excluded from analyses as practice. Of the remaining trials, $9.6 \%$ of the trials were terminated abruptly because gaze position was outside the fixation criterion before target onset. Again, because these trials contain no information about saccadic responses to target onsets, these trials were excluded from analyses. There were thus 3,744 total trials.

Saccadic eye movement errors away from the target were rare (see Table 1), but the error rates for cued trials relative to the uncued locations on central arrow trials in the mixed target condition exceeded Fisher's least significant difference $(.023$, or $2.30 \%)$, revealing more errors for cued trials. Similarly, anticipations were rare but higher than in Experiment 1, occurring on $1.81 \%^{2}$ of the trials. SRTs in excess of $1 \mathrm{~s}$ occurred in $0.43 \%$ of the trials. These data were excluded from analyses. The SRTs and error rates can be found in Table 1.

The remaining 3,619 trials were submitted to a 2 (cued or uncued target) $\times 2$ (central or peripheral target) repeated measures ANOVA. The rightmost panel in Fig. 5 shows the magnitude of IOR for centrally and peripherally presented targets. There was a main effect of target type, $F(1,12)=$ $37.73, p<.05$, showing shorter SRTs for peripheral $(M=$ $235.49 \mathrm{~ms})$ than for central $(M=293.21 \mathrm{~ms})$ targets. There was a main effect of cuing, $\mathrm{F}(1,12)=37.84, p<.05$, revealing IOR. Critically, the interaction between cuing and target type was not significant, $\mathrm{F}<1$, demonstrating that IOR was equivalent for central $(M=27.22 \mathrm{~ms})$ and peripheral $(M=$ $28.18 \mathrm{~ms})$ targets.

\section{Discussion}

Experiment 2 was conducted to determine whether the fixation removal in the mixed condition of Experiment 1 with peripheral targets might have lowered the true IOR score in this condition, thus obscuring an input component of IOR. It

\footnotetext{
${ }^{2}$ One participant contributed 32 anticipations (and had an accuracy of $50 \%$ on these trials) to the data set, which, in large part, explains the numerical differences in anticipation rates between the mixed condition in Experiment 1 and the present experiment. It should be noted that this participant's accuracy was at $98 \%$ when anticipations were excluded from analysis.
} 
had not. As in Taylor and Klein (2000), we obtained equivalent IOR for peripheral and central targets when target onset and the fixation stimulus overlapped.

\section{General discussion}

Considered together, our two experiments demonstrate that neither perceptual confusion nor the fixation removal confound in Abrams and Dobkin (1994b) were responsible for their pattern of results being different from the pattern in Taylor and Klein (2000) in comparable conditions (see Fig. 2). In both experiments, we used the same potentially perceptually confusing stimulus sequence as did Abrams and Dobkin (1994b), and yet, when central and peripheral targets were mixed in a block, we found equivalent IOR in these conditions. And when these target types were mixed, whether we removed fixation at the time of the target in the peripheral but not in the central condition (Experiment 1), as did Abrams and Dobkin (1994b), or we eliminated this confound (Experiment 2), IOR with the two target types was statistically equivalent. Only when we administered the two target types in separate blocks did we find greater IOR with peripheral than with central targets (indeed, we found no evidence for IOR where central targets were blocked).

Our demonstration that mixing versus blocking the type of target was responsible for the discrepancy between Abrams and Dobkin (1994b) and Taylor and Klein (2000) has an important methodological message. When a block of trials is made up entirely of centrally presented targets, a spatial ACS can be established to discount irrelevant inputs at nontarget locations (namely, the peripheral cues in the present experiment) and, consequently, reduce or eliminate the IOR such cues might otherwise have generated (cf. Wang \& Klein, 2012). As such, to the extent that the spatial ACS is effectively implemented, little (Abrams \& Dobkin, 1994b; Rafal et al., 1994) to no (Li \& Lin, 2002; the present Experiment 1) IOR will be generated by cues that never appear at the same location(s) as the targets. The blocking of conditions generally - and more specifically, the type of imperative stimulus - allows the participant to establish, in advance of each trial, a mental set specifically designed for the upcoming conditions (targets). When one is seeking to demonstrate or to explore the nature of such strategic adaptations (as was done by Folk et al., 1992), blocking is the method of choice. However, when one is interested in comparing, on a "level playing field," effect magnitudes across these conditions, blocking is - as has been explicitly suggested by Jonides and Mack (1984) and implied by numerous investigations that have compared mixing versus blocking to reveal the strategic nature of differences in the blocked paradigm (e.g., Klein, 1977; Pratt et al., 2001)- untoward: Blocking allows strategic differences to be confounded with the factor that is blocked.

When we avoided such a strategy confound by mixing target types, we found that the magnitude of IOR measured by exogenously and endogenously generated saccades was equivalent. The theoretical implication of this finding (see Fig. 1) is that there is a single, motoric effect of oculomotor IOR (Taylor \& Klein, 1998, 2000) when the effect of a tobe-ignored peripheral onset is measured $1 \mathrm{~s}$ later by saccadic responses to previously stimulated locations. Simply, saccadic responses are slowed by comparable magnitudes to regions that have been previously stimulated, irrespective of whether the imperative saccade-generating stimulus occurs there or elsewhere. If one effect of oculomotor IOR were to degrade the input signal at a cued location in a no-responsesaccade paradigm, the magnitude of IOR would have been greater in the mixed condition for peripheral targets relative to central targets, a result that is conspicuously absent when the ACS for the cue is equated between target presentation conditions. Therefore, these findings rule strongly against the idea of a perceptual/attentional effect to oculomotor IOR and against most two-component theories postulating an attentional/perceptual component in addition to a motoric component (Abrams \& Dobkin, 1994b; Fecteau, Au, Armstrong, \& Munoz, 2004; Khatoon, Briand, \& Sereno, 2002; Li \& Lin, 2002; Rafal et al., 1994; Sereno, Jeter, Pariyadath, \& Briand, 2006).

Were there transient, sequence-induced attentional control settings?

We wondered whether the kind of ACS we have hypothesized that participants adopt when they are faced with a block of trials with all targets at fixation or in the periphery might be subject to much shorter-term influences from the previous trial in a mixed block. For example, in his study of global/local processing, Ward (1982) concluded that "processing is faster at a given level if previous processing has been at that level." We wanted to see whether such a sequential effect might be operating when the previous trial entailed processing an imperative signal at fixation or in the periphery. Examination of sequential effects requires the exclusion of all two-trial sequences that includes an error or inappropriate eye movement. In order to overcome the large loss of data this exclusion entails, we subjected the mixed block data from both Experiments 1 and 2 to analysis. In this analysis, there were no significant main effects or interactions involving experiment. As was expected, the main effect of cuing (IOR) was significant, and cuing was unaffected by the present trial's target location. Importantly, the prior trial's target location (central vs. peripheral) had no effect on the current trial's cuing effect, $F<1$, nor did it contribute to any significant interactions. 
Implications for the literature

Although the principal aim of this study was to determine whether evidence for two components of IOR can be obtained when processing of the cue is balanced by way of a mixed design, the ACS hypothesis carries important implications for the IOR literature and calls for a reevaluation of several findings. This point is best illustrated with examples.

Consider Li and Lin (2002). In their "vertically instructed saccade" paradigm, there were four placeholder locations equidistant from fixation. Two placeholders appeared left and right, and two placeholders appeared above and below fixation. The cue, a peripheral onset, appeared in either the left or right placeholder location. The "target," however, appeared at one of the two locations on the vertical axis. A target at the top placeholder location on the vertical axis signaled a rightward saccade, and a target at the bottom placeholder location signaled a leftward saccade. In this paradigm, there was no difference in RTs to saccades directed at previously cued and uncued locations, which was interpreted as evidence against any motor view of IOR (component or flavor as described here). In light of the present finding, and according to ACS theory, however, it seems likely that the absence of IOR occurred, not because there is no motor mechanism, but rather because participants had adopted an attentional set to focus on the vertical locations where the targets were presented (see the section in the General Discussion on antisaccades for an alternative explanation). Consequently, the task-irrelevant horizontal cues were effectively filtered out, generating no IOR (see Wang \& Klein, 2012, for direct evidence that such an effect is possible when attention is focused strongly at fixation when the peripheral cues are presented).

Next, we will consider several findings that have explored the dynamic remapping of IOR in variations on the no-response-saccade paradigm (Abrams \& Dobkin, 1994b; Abrams \& Pratt, 2000). Follow-up studies in Abrams and Dobkin (1994b, Experiments 3 and 4) attempted to determine whether the "motor and attentional component IOR" mapped in object-centered coordinates (cf. Tipper, Driver, \& Weaver, 1991, for evidence of object-based coding of IOR in a no-response-manual paradigm). The sequence of events prior to target onset was similar to what is shown in Fig. 4, except for the following: (1) The placeholder boxes were oriented vertically to begin the trial; (2) the boxes rotated smoothly $90^{\circ}$ clockwise after the delivery of the asterisk stimulus at fixation; and (3) central and peripheral targets were presented in separate blocks to different groups. The key result here was that a dynamic reference frame of IOR was found when measured by peripheral targets (in Experiment 3,14.3 ms of IOR was observed), but not central targets (in Experiment 4, $2.5 \mathrm{~ms}$ of facilitation was observed). This led Abrams and Dobkin (1994b) to conclude that the attentional/perceptual component of IOR was object centered, whereas the motoric component was retinotopic. A more reasonable interpretation, given the present results, might simply be that observers were successfully ignoring the cue in the central arrow target condition.

Similarly, Abrams and Pratt (2000) examined whether the coordinate system of oculomotor IOR was environmental or retinotopic. In this case, IOR was again measured by a central arrow stimulus, but much as in Abrams and Dobkin (1994b), task-relevant stimuli never appeared at cued locations. Here, a weak $(\sim 5-\mathrm{ms})$ retinotopic IOR effect was found, whereas IOR did not exist at the spatiotopic location. This failure to find IOR in spatiotopic coordinates is particularly suspicious, given that oculomotor IOR is commonly found to exist in a dynamic reference frame during search of simple (cf. Gilchrist \& Harvey, 2000; Mathot \& Theeuwes, 2011; Peterson, Kramer, Wang, Irwin, \& McCarley, 2001) and complex (cf. Hofler, Gilchrist, \& Korner, 2011; MacInnes \& Klein, 2003; Smith \& Henderson, in press) visual displays (for reviews, see Reppa, Schmidt, \& Leek, 2012; Wang \& Klein, 2010). Evidently, the failure to show a dynamic remapping for oculomotor IOR when measured by a centrally presented arrow needs to be reexamined in light of the present findings.

The conclusions in the literature from results like those discussed in this section must be reconsidered, and we hope that these examples will encourage a dedicated exploration of other studies.

\section{Conclusions}

We found that blocking target type leads to significantly more IOR for peripheral relative to central targets with Abrams and Dobkin's (1994b) methods. Mixing target types, while preserving other methodological features of Abrams and Dobkin (1994b), eliminates this difference. And, when the fixation removal confound linked with central versus peripheral cuing that characterized Abrams and Dobkin's (1994b) methods was eliminated, the equality of IOR following central and peripheral cues, as reported by Taylor and Klein (2000, Experiment 4) was preserved. The difference in the magnitude of IOR in the blocked design is most likely due to an attentional control setting, or "attentional window," that is adjusted to encompass the locations where behaviorally relevant stimuli (targets) are presented. In a block with only central arrow targets, the window could be narrowly focused around fixation; in a block containing peripheral targets, the window would be widened to include those peripheral locations. This difference in the distribution of attention prior to cue onset modulates the magnitude of IOR that is generated by the cues (cf. Wang \& Klein, 2012). 
That IOR is equivalent for peripherally and centrally presented targets when the distribution of attention prior to the cue for central and peripheral targets strongly suggests that IOR is not attentional/perceptual when measured in a noresponse (to a peripheral onset)-prosaccade (to a peripheral onset or central arrow) paradigm. Following the logic (see Fig. 1) of Abrams and Dobkin (1994b), our results strongly suggest that under conditions where IOR is caused by an uninformative peripheral event and measured by a saccade to a peripheral event (or to a central arrow) presented $1 \mathrm{~s}$ later, the effect of IOR (Posner et al., 1985) is near the output end of the processing continuum.

\section{References}

Abrams, R. A., \& Dobkin, R. S. (1994a). The gap effect and inhibition of return: Interactive effects on eye movement latencies. Experimental Brain Research, 98, 483-487.

Abrams, R. A., \& Dobkin, R. S. (1994b). Inhibition of return: Effects of attentional cuing on eye movement latencies. Journal of Experimental Psychology. Human Perception and Performance, 20, 467-477.

Abrams, R. A., \& Pratt, J. (2000). Oculocentric coding of inhibited eye movements to recently attended locations. Journal of Experimental Psychology. Human Perception and Performance, 26, 776788

Chica, A. B., Taylor, T. L., Lupiáñez, J., \& Klein, R. M. (2010). Two mechanisms underlying inhibition of return. Experimental Brain Research, 201, 25-35.

Eriksen, C. W., \& St. James, J. D. (1986). Visual attention within and around the field of focal attention: A zoom-lens model. Perception \& Psychophysics, 40, 225-240.

Fecteau, J. H., Au, C., Armstrong, I. T., \& Munoz, D. P. (2004). Sensory biases produce alternation advantage found in sequential saccadic eye movement tasks. Experimental Brain Research, 159, 84-91.

Folk, C. L., Remington, R. W., \& Johnston, J. C. (1992). Involuntary covert orienting is contingent on attentional control settings. Journal of Experimental Psychology. Human Perception and Performance, 18, 1030-1044.

Gibson, B. S., \& Amelio, J. (2000). Inhibition of return and attentional control settings. Perception \& Psychophysics, 62, 496-504.

Gilchrist, I. D., \& Harvey, M. (2000). Refixation frequency and memory mechanisms in visual search. Current Biology, 10, 12091212.

Goldsmith, M., \& Yeari, M. (2003). Modulation of object-based attention by spatial focus under endogenous and exogenous orienting. Journal of Experimental Psychology. Human Perception and Performance, 29, 897-918.

Guimaraes-Silva, S., Gawryszewski, L. G., Portugal, T. S., \& Klausner-de-Oliveira, L. (2004). Inhibition of return, gap effect and saccadic reaction time to a visual target. Brazilian Journal of Medical and Biological Research, 37, 533-538.

Hecht, S., Schlaer, S., \& Pirenne, M. H. (1942). Energy, quanta, and vision. The Journal of General Physiology, 42, 819-840.

Hofler, M., Gilchrist, I. D., \& Korner, C. (2011). Inhibition of return functions within but not across searches. Attention, Perception, \& Psychophysics, 73, 1385-1397.
Hunt, A. R., \& Kingstone, A. (2003). Inhibition of return: Dissociating attentional and oculomotor components. Journal of Experimental Psychology. Human Perception and Performance, 29, 1068-1074.

Ishigami, Y., Klein, R. M., \& Christie, J. (2009). Using illusory line motion to explore attentional capture. Visual Cognition, 17, 431-456.

Jonides, J., \& Mack, R. (1984). On the cost and benefit of cost and benefit. Psychological Bulletin, 96, 29-44.

Khatoon, S., Briand, K. A., \& Sereno, A. B. (2002). The role of response in spatial attention: Direct versus indirect stimulus-response mappings. Vision Research, 42, 2693-2708.

Kingstone, A., \& Klein, R. M. (1993). What are human express saccades. Perception \& Psychophysics, 54, 260-273.

Kingstone, A., \& Pratt, J. (1999). Inhibition of return is composed of attentional and oculomotor processes. Perception \& Psychophysics, 61, 1046-1054.

Klein, R. M. (1977). Attention and visual dominance: A chronometric analysis. Journal of Experimental Psychology. Human Perception and Performance, 3, 365-378.

Klein, R. M. (2000). Inhibition of return. Trends in Cognitive Sciences, 4, 138-147.

Klein, R. M. (2005). On the role of endogenous orienting in the inhibitory aftermath of exogenous orienting. In U. Mayr, E. Awh, \& S. W. Keele (Eds.), Developing individuality in the human brain: A tribute to Michael I. Posner (pp. 45-64). Washington, DC: American Psychological Association.

Klein, R. M., \& Hilchey, M. D. (2011). Oculomotor IOR. In I. Gilchrist, I. D. Liversedge, \& S. Everling (Eds.), Oxford handbook on eye movements (pp. 471-492). Oxford: Oxford University Press.

Laberge, D. (1983). Spatial extent of attention to letters and words. Journal of Experimental Psychology. Human Perception and Performance, 9, 371-379.

Li, C. S. R., \& Lin, S. C. (2002). A perceptual level mechanism of the inhibition of return in oculomotor planning. Cognitive Brain Research, 14, 269-276.

Lupiáñez, J., \& Milliken, B. (1999). Inhibition of return and the attentional set for integrating versus differentiating information. The Journal of General Psychology, 126, 392-418.

MacInnes, J. W., \& Klein, R. M. (2003). Inhibition of return biases orienting during the search of Posner complex scenes. The Scientific World Journal, 3, 75-86.

Mathot, S., \& Theeuwes, J. (2011). Gradual remapping results in early retinotopic and late spatiotopic inhibition of return. Psychological Science, 21, 1793-1798.

Peterson, M. S., Kramer, A. F., Wang, R. F., Irwin, D. E., \& McCarley, J. S. (2001). Visual search has memory. Psychological Science, 12, 287-292.

Posner, M. I., \& Cohen, Y. (1984). Components of visual orienting. In H. Bouma \& D. G. Bouwhuis (Eds.), Attention and performance $X$ : Control of language processes (pp. 531-556). Hove, UK: Erlbaum.

Posner, M. I., Rafal, R. D., Choate, L., \& Vaughan, J. (1985). Inhibition of return: Neural basis and function. Cognitive Neuropsychologia, 2, 211-228.

Pratt, J., \& McAuliffe, J. (2002). Determining whether attentional control settings are inclusive or exclusive. Perception \& Psychophysics, 64, 1361-1370.

Pratt, J., \& Neggers, B. (2008). Inhibition of return in single and dual tasks: Examining saccadic, keypress, and pointing responses. Perception \& Psychophysics, 70, 257-265.

Pratt, J., Sekuler, A. B., \& McAuliffe, J. (2001). The role of attentional set on attentional cueing and inhibition of return. Visual Cognition, 8, 33-46.

Rafal, R. D., Egly, R., \& Rhodes, D. (1994). Effects of inhibition of return on voluntary and visually guided saccades. Canadian Journal of Experimental Psychology, 48, 284-300. 
Reppa, I., Schmidt, W. C., \& Leek, E. C. (2012). Successes and failures in producing attentional object-based cueing effects. Attention, Perception, \& Psychophysics, 74, 43-69.

Reuter-Lorenz, P. A., Jha, A. P., \& Rosenquist, J. N. (1996). What is inhibited in inhibition of return? Journal of Experimental Psychology. Human Perception and Performance, 22, 367-378.

Samuel, A. G., \& Kat, D. (2003). Inhibition of return: A graphical meta-analysis of its time course and an empirical test of its temporal and spatial properties. Psychonomic Bulletin \& Review, 10, 897-906.

Sereno, A. B., Jeter, C. B., Pariyadath, V., \& Briand, K. A. (2006). Dissociating sensory and motor components of inhibition of return. The Scientific World Journal, 6, 862-887.

Smith, T. J., \& Henderson, J. M. (in press). Does oculomotor inhibition of return influence fixation probability during scene search? Attention, Perception, \& Psychophysics. doi:10.3758/s13414-011-0191-x

Souto, D., \& Kerzel, D. (2009). Evidence for an attentional component in saccadic inhibition of return. Experimental Brain Research, 195, 531-540.

Taylor, T. L., \& Klein, R. M. (1998). On the causes and effects of inhibition of return. Psychonomic Bulletin \& Review, 5, 625-643.
Taylor, T. L., \& Klein, R. M. (2000). Visual and motor effects in inhibition of return. Journal of Experimental Psychology. Human Perception and Performance, 26, 1639-1656.

Theeuwes, J. (1991). Exogenous and endogenous control of attention: The effect of visual onsets and offsets. Perception \& Psychophysics, 49, 83-90.

Theeuwes, J. (1994). Exogenous and endogenous control of attention: The effect of visual onsets and offsets. Perception, 23, 429-440.

Tipper, S. P., Driver, J., \& Weaver, B. (1991). Object-centered inhibition of return of visual attention. Quarterly Journal of Experimental Psychology, 43A, 289-298.

Wang, Z., \& Klein, R. M. (2010). Searching for inhibition of return in visual search: A review. Vision Research, 50, 220-228.

Wang, Z., \& Klein, R. M. (2012). Focal spatial attention eliminates inhibition of return. Psychonomic Bulletin \& Review, 3, 462-469.

Ward, L. W. (1982). Determinants of attention to local and global features of visual forms. Journal of Experimental Psychology. Human Perception and Performance, 8, 562-581.

Yantis, S., \& Jonides, J. (1990). Abrupt visual onsets and selective attention - voluntary versus automatic allocation. Journal of Experimental Psychology. Human Perception and Performance, 16, 121-134. 